\title{
An Optimal LED Light Source Arrangement to Reduce SNR Fluctuation for MISO VLC System Using Fireworks Algorithm
}

\author{
Chunxia You ${ }^{1,2}$, Shen Zhang ${ }^{1, *}$, Ran Zhang ${ }^{1}$ \\ 1. The Internet of Things Research Center, China University of Mining and \\ Technology, Xuzhou, China. \\ 2. School of Physics and Electronic Engineering, Jiangsu Normal University, \\ Xuzhou, China. \\ Iyoucx@jsnu.edu.cn, ${ }^{2 *}$ zhangshencumt@163.com, 452287268@qq.com
}

\begin{abstract}
This paper investigates a novel LED lamp arrangement to reduce SNR fluctuation in indoor visible light communication system. In VLC system, the signal sources often come from several LED lamps. The different positions of the LED lamps will influence the system quality. In our proposed scheme, the LED lamp arrangement consists of $N$ LED lamps spreading in the ceiling. The heights of all lamps are the same of 3 meters which is the height of communication room. An optimal LED arrangement with the firework algorithm is presented for the minimum SNR factor and SNR fluctuation on the light receiver plane no matter the number of lamps. In order to compare the effect of SNR distributions, the total power of N LED lamps is identical. Our studies show that the SNR factor under such a LED arrangement is reduced from 118.5 to 4.09 with 16 LED lamps multi-input system which effectively reduces the SNR fluctuation between different users. Such arrangement guarantees all users obtain almost identical communication quality regardless of users' locations.
\end{abstract}

Keywords: visible light communications, Light-emitting diodes, Fireworks algorithm, optimal LED arrangement, optical wireless communications

\section{Introduction}

Visible light communication (VLC), with obvious characters of high transmission rate and free spectrum for wireless services especially in indoor environments, has been proposed as a promising wireless communication technology in the recent years [1-5]. In indoor VLC systems, LED lamps are usually located in the center or evenly separated with the style of matrix arrays in the ceiling of the room. These arrangements result in intensive fluctuations of the signal to noise ratio (SNR) on the receiver plane, which causes that users acquire various quality signals for their different locations. Intensive SNR fluctuations will increase the complexity of VLC system to guarantee the same communication quality between different users, especially user in the corner or edge of the communication space. In order to solve this problem, some LED arrangements have been proposed in [1, 6-7]. 12 LED lamps spread evenly on a circle and 4 LED lamps positioned in the four corners to reduce SNR fluctuation between different users in reference [6]. The uniform matrix distribution is adopted in [1] and LED lamp powers are adjusted to reduce the SNR fluctuation in [7]. To our knowledge, there isn't an article to adaptively regulate the LED lamp position for the same quality receiver signal of multiusers. In this paper, we search the optimal position of every LED lamp by applying fireworks algorithm in the room ceiling to get identical quality received signals. To evaluate the SNR fluctuation on the receiver plane, the SNR factor is introduced. The smaller SNR factor means the smaller SNR fluctuation and the better SNR 
distribution of received signal. Such optimal LED arrangement can decrease the SNR factor from 118.5 to 4.09 in a 16 light sources VLC system and to 31.4 when the number of lamps is 4 . So, the same quality signals can be received without more complicated receivers under such optimal LED arrangement method.

The body of this paper is organized as follows. Section 2 describes the VLC system model and introduces the optimal theory for minimize SNR factor. In section 3, the optimal LED lamps arrangement is processed by fireworks algorithm. Section 4 shows simulation results under our proposed optimal lamps arrangement. Section 5 concludes the paper.

\section{System Model and Optimal Theory}

In VLC system, we use the common white LED whose radiant irradiance is obeyed Lambertian radiation pattern :

$I\left(\varphi_{i}\right)=\frac{(m+1) \cos ^{m}\left(\varphi_{i}\right)}{2 \pi}$,

where $m$ is the order of Lambertian emission defined as $m=-\ln 2 / \ln \left(\cos \left(\varphi_{1 / 2}\right)\right)$, $\varphi_{1 / 2}$ is the semi-angle at half power of the LED lamp, $\varphi_{i}$ is the angle of irradiance from the i-th LED lamp as shown in figure 1. In our system, all LED lamps have the same type. The channel direct current (DC) gain between the i-th lamp and one receiver is described by $[1,8]$

$H_{i}(0)=\frac{I\left(\varphi_{i}\right) \cos \left(\theta_{i}\right) A}{L_{i}^{2}}=\frac{(m+1) \cos ^{m}\left(\varphi_{i}\right) A \cos \left(\theta_{i}\right)}{2 \pi L_{i}^{2}}$,

where $L_{i}$ is the distance between i-th LED lamp and the receiver, $A$ is the light contact area of the photo-detector, $\theta_{i}$ is the angle of incidence. Note that in this paper, all LED lamps and photo-detectors are horizontally placed.

The horizontal illumination $E_{i}$ caused by the i-th LED lamp is given by

$E_{i}=\frac{(m+1)}{2 \pi L_{i}^{2}} \cos ^{m}\left(\varphi_{i}\right) * \cos \left(\theta_{i}\right)$.

There is a total of N LED lamps as light sources. So, the whole illumination $E$ is given as $E=\sum_{i=1}^{N} E_{i}$ at a receiver point and $E_{i}$ is the illumination caused by i-th lamp.

In this paper, only line of sight signals are considered. Then, the total power $P_{r}$ on a receiver is given as $P_{r}=\sum_{i=1}^{N} H_{i}(0) P_{t}$ from these $N$ LED lamps. $N$ is the number of light signal sources. $P_{t}$ is the transmit power of LED lamp. Note that, the value of every LED lamp power is a constant and the total value of lamps power is 80 watts. So, when the number of LED lamps is $N$, the power of every lamp is $80 / N$ watts. 


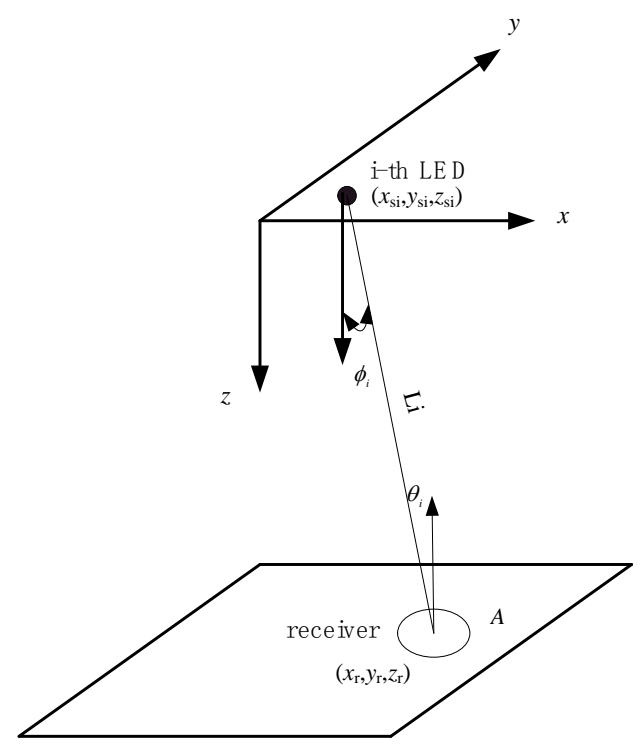

Figure 1. Geometry of the i-th LED and a Receiver with Line of Sight

The LED sources and the receivers are horizontal. So, the angle of incidence $\theta_{i}$ are equal to the angle of irradiance $\varphi_{i}$ which are described as $\theta_{i}=\arccos \frac{\left|z_{r}-z_{s i}\right|}{L_{i}}$

$\theta_{i}=\arccos \frac{\left|z_{r}-z_{s i}\right|}{\sqrt{\left(x_{r}-\mathrm{x}_{\mathrm{si}}\right)^{2}+\left(y_{r}-\mathrm{y}_{\mathrm{si}}\right)^{2}+\left(z_{r}-\mathrm{z}_{\mathrm{si}}\right)^{2}}}$.

$\left(x_{s i}, y_{s i}, z_{s i}\right)$ is the three-dimensional coordinate of the i-th LED and $\left(x_{r}, y_{r}, z_{r}\right)$ is the three dimensional coordinate of receiver.

At the receiver, the mixed signal to noise ratio (SNR) from $\mathrm{N}$ lamps is described as

$$
S N R_{j}=\frac{\overline{s^{2}(t)}}{P_{\text {nose }}}=\frac{\sum_{i=1}^{N}\left(R H_{i}(0) P_{t} M_{I}\right)^{2} \overline{f^{2}(t)}}{R_{b} N_{0}},
$$

Where $P_{\text {noise }}$ is the power of noise which is defined in [1]. $\overline{s^{2}(t)}$ is the signal average power of the output detected electrical signal. $\mathrm{R}$ is the responsivity of the photo detector. $M_{I}$ is the modulation index. The noise is assumed as an additive white Gaussian noise and the power is described as $R_{b} N_{0}$, where $R_{b}$ is the data rate of the system and $N_{0}$ is the variance of white noise.

To evaluate the SNR fluctuation and compute the optimal situations of LED lamps based on the firework algorithm, we introduce the SNR factor to evaluate the whole SNR distribution of all receiver points, which is described as

$$
Q_{S N R}\left(x_{s 1}, y_{s 1}, \ldots, x_{s N}, y_{s N}\right)=\frac{\sqrt{\operatorname{var}(S N R)}}{\overline{S N R}},
$$

where $\operatorname{var}(\mathrm{SNR})$ is the variance of all receiver points SNR and $\overline{S N R}$ is the mean value of SNR. When the SNR variance is smaller and the mean SNR is bigger, the smaller SNR factor will be received which means the more uniform SNR distribution on the receiver plane. In theory, the smallest SNR factor is zero which means all users have the same signal quality. In fact, we can't get the accurate identical quality signal. So, we optimize situations of LED lamps to get the enough small SNR factor. Note that the reflection by 
walls isn't considered in the following analyses. There are $\mathrm{N}$ identical LED lamps distributed in the ceiling with 3 meter height as multiple input signals in system. We sample one position every 5 centimeters on the receiver plane where the height is 0.85 meter. The total power of $\mathrm{N}$ LEDs is $\mathrm{N}^{*} \mathrm{Pt}$ watts in different LED arrangement for comparing the SNR distribution.

In the system, we set $\mathrm{N}$ LED lamp positions as $S_{i}, i=1,2, \ldots, N$. Every light source position has a three-dimensional coordinate with $S_{i}\left(x_{s i}, y_{s i}, z_{s i}\right) i=1,2, \ldots, N$. The height $z_{s i}$ of each light is 3 meters constant. It is just the height of the room.

The problem is adjusting the position vector $P\left(x_{s 1}, y_{s 1}, \ldots, x_{s N}, y_{s N}\right)$ to minimize the $Q_{S N R}$

minimize $Q_{S N R}\left(x_{s 1}, y_{s 1}, \ldots, x_{s N}, y_{s N}\right)$.

The ideal $Q_{S N R}$ is value 0 . In fact, no vector P can achieve this perfect $Q_{S N R}$ value. So, when $Q_{S N R} \leq \gamma$, where $\gamma$ is a small constant which we can adopt, the position vector $\mathrm{P}$ is considered to be received.

\section{Positions Optimization based on the Fireworks Algorithm}

In this section, the optimal positions vector $P$ is searched based on the intelligent fireworks algorithm. Vector $P$ means the optimal coordinate of $N_{t}$ LED lamps. And the minimal SNR factor can be received under this lamp arrangement. The fireworks algorithm is a novel intelligence algorithm proposed for function optimization in $[9,10]$. It simulates the explosions of fireworks to search the global optimum value. When a firework is set off, a shower of sparks will fill around it. The explosion process of a firework is viewed as a search for a specific point $[11,12]$. To keep diversity of sparks, two explosions are employed. So, the fireworks algorithm can avoid the local optimum value and get the global optimum value in the searching progress.

In the optimization progress, the SNR factor $Q_{S N R}\left(x_{s i}, y_{s i}\right)$ is set as the target function. $P\left(x_{s 1}, y_{s 1}, \ldots, x_{s N}, y_{s N}\right)$ compose of a $2 \mathrm{~N}$-dimensional parameter as a space position for fireworks algorithm. The optimal process is designed to optimize positions for the problem:

Minimize $Q_{S N R}\left(x_{s 1}, y_{s 1}, \ldots, x_{s N}, y_{s N}\right) i=1,2, \ldots, N_{t} \in \mathfrak{R}, \quad 0 \leq x_{s i} \leq x_{\max }, 0 \leq y_{s i} \leq y_{\max }$,

where $x_{\max }$ and $y_{\max }$ are the maximum abscissa and ordinate value which are the length and width of the room in communication space.

The optimal framework is shown as figure 2. The initial fireworks locations $p_{1}, p_{2} \ldots . . p_{n}$ are randomly selected at first. The number of fireworks is $\mathrm{n}$. Every firework location is a $2 \mathrm{~N}$-dimension data. This $2 \mathrm{~N}$-dimension parameter is a space position in fireworks algorithm. Then, every firework explodes some sparks. The number of sparks generated by a firework is described as:

$s_{i}=m \cdot \frac{Q_{\max }-Q\left(p_{i}\right)+\zeta}{\sum_{i=1}^{n}\left(Q_{\max }-Q\left(p_{i}\right)\right)+\zeta}$,

where $\mathrm{m}$ is a parameter for controlling the total number of sparks generated by fireworks, $Q_{\max }=\max \left(Q_{S \backslash R}\left(p_{i}\right)\right) \quad(i=1,2, \ldots, n)$ is the maximum value of the objective function, $Q\left(p_{i}\right)$ is the objective function value of the location $p_{i}$, and $\zeta$ is the smallest constant to avoid zero-division-error in computer count.

A spark is generated as following:

(1) Initialize the spark location: $\tilde{p}_{j}=p_{i}$; 
(2) Generate a random number z: $z=\operatorname{round}(d \bullet \operatorname{rand}(0,1))$, where $\mathrm{d}$ is the dimensionality of the space location ;

(3) Select random z dimensions of $\tilde{p}_{j}$;

(4) Calculate the amplitude of explosion $A_{i}: A_{i}=\hat{A} \bullet \frac{Q\left(p_{i}\right)-Q_{\min }+\zeta}{\sum_{i=1}^{n}\left(Q\left(p_{i}\right)-Q_{\min }\right)+\zeta}$, where $\hat{A}$ is the maximum explosion amplitude and $Q_{\min }=\min \left(Q_{S N R}\left(p_{i}\right)\right) \quad(i=1,2, \ldots, n)$ is the minimum value of the objective function;

(5) Calculate the displacement: $h=A_{i} \bullet \operatorname{rand}(0,1)$;

(6) For pre-selected z dimensions do $\tilde{p}_{k}^{j}=\tilde{p}_{k}^{j}+h \quad k=1,2, \ldots, z$;

(7) If $\tilde{p}_{k}^{j}<0$ or $\tilde{p}_{k}^{j}>5$ then $\tilde{p}_{k}^{j}=\tilde{p}_{k}^{\min }+\left|\tilde{p}_{k}^{j}\right| \%\left(p_{k}^{\max }-p_{k}^{\min }\right)$;

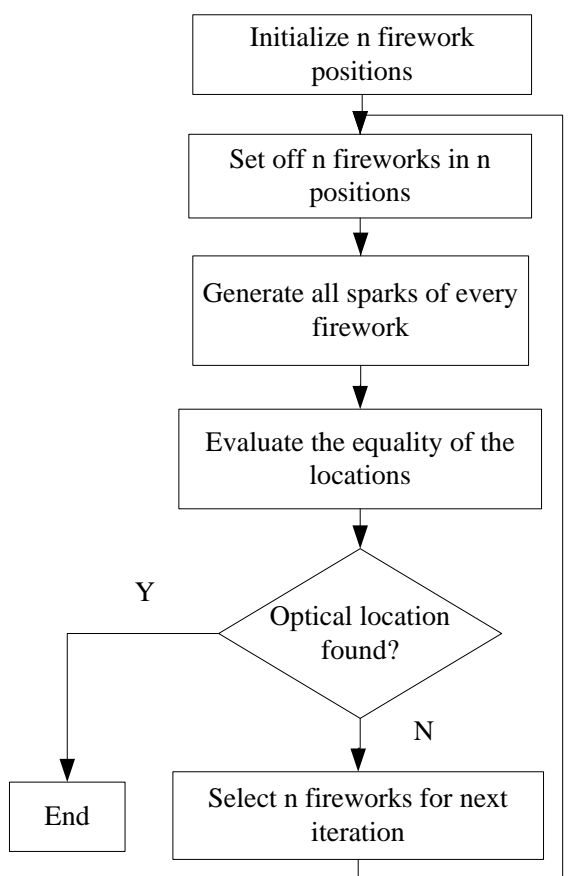

\section{Figure 2. Framework of Position Optimization based on Fireworks Algorithm}

For keeping the diversity of sparks, Gaussian explosion sparks are designed. Replace (5) and (6) with the Gaussian coefficient $g=$ Gaussian(1,1) and $\tilde{p}_{k}^{j}=\tilde{p}_{k}^{j} \bullet g \quad k=1,2, \ldots, z$. So, a Gaussian explosion sparks can be received.

With this method, all sparks are received for $\mathrm{n}$ fireworks. An optimal location is selected from all fireworks and sparks as one firework for the next generation iteration, whose object function $Q_{S N R}$ has the minimum value. In the next iteration, the rest $\mathrm{n}-1$ fireworks are selected from all remaining fireworks and sparks with probability::

$P\left(p_{i}\right)=\frac{R\left(p_{i}\right)}{\sum_{j \in K} R\left(p_{j}\right)}$ 
$P\left(p_{i}\right)$ is the probability of $p_{i}$ as the next firework. The distance between one location $p_{i}$ and other locations is described as:

$$
R\left(p_{i}\right)=\sum_{j \in K} d\left(p_{i}, p_{j}\right)=\sum_{j \in K}\left\|p_{i}-p_{j}\right\|,
$$

where $\mathrm{K}$ is the set of all current locations of both fireworks and sparks. If the iteration number reach the maximum value or the object function value reach the minimum target value which we set, the program ends and the optimal progress is accomplished.

\section{Simulation Results}

In this section, we analyze the performance of our proposed optimal LED lamp arrangement with simulation. All programs and results in this paper are produced by MATLAB software. Note that, if the number $N$ is 16 , there are 16 LED lamps as signal transmitting equipments. 16 abscissa parameters and 16 ordinate parameters for 16 LED lights will be optimized. The room size (length $\times$ width $\times$ height) is $5 \mathrm{~m} \times 5 \mathrm{~m} \times 3 \mathrm{~m}$. The height of desk where the receiver is located is $0.85 \mathrm{~m}$. The total value of $N$ lamp powers is 80 watts. The data transmitting rate $R_{b}$ is $10 \mathrm{Mbps}$. The other parameters are listed in Table 1.

Table 1. Parameters of VIC System Configuration

\begin{tabular}{|c|c|}
\hline Length of room & $5 \mathrm{~m}$ \\
\hline Width of room & $5 \mathrm{~m}$ \\
\hline Height of room & $3 \mathrm{~m}$ \\
\hline $\begin{array}{c}\text { Height of plane where the } \\
\text { receiver is located }\end{array}$ & $0.85 \mathrm{~m}$ \\
\hline $\begin{array}{c}\text { Transmitter's semi-angle at half } \\
\text { power }\end{array}$ & $60^{\circ}$ \\
\hline Physical area of photo-detector & $10^{-4} \mathrm{~m}^{2}$ \\
\hline $\begin{array}{c}\text { Responsivity of photo } \\
\text { detector }(R)\end{array}$ & $1 \mathrm{~A} / \mathrm{W}$ \\
\hline Modulation index ( $\left.M_{I}\right)$ & 0.2 \\
\hline Average power of signal & $1 \mathrm{~W}$ \\
\hline Total power of N lamps & $80 \mathrm{~W}$ \\
\hline Data transmission rate $R_{b}$ & $10 \mathrm{Mbps}$ \\
\hline
\end{tabular}

In fireworks algorithm, fireworks are randomly generated in the beginning progress of optimization. For decreasing the iteration number and increasing the iteration rate, we initial the $\mathrm{N}$ lamps spread evenly in the ceiling with uniform matrix. As shown in figure 3, when $\mathrm{N}$ equals 16 , the initial abscissa of these lamps are respectively $0.625,1.875,3.125$, 4.375 and the ordinate of these lamps are respectively $0.625,1.875,3.125,4.375$ instead of random positions. When the total LED number is 4 , the initial abscissa of these lamps are respectively 1.253 .75 and the ordinate of these lamps are respectively 1.25 3.75. 


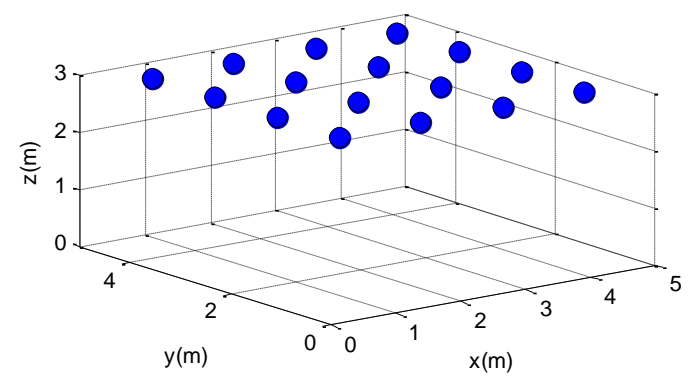

Figure 3. Initial Positions of 16 LED Lamps

The iteration number comparison of two different initial positions in fireworks algorithm is shown in figure 4 when $\mathrm{N}$ equals 16 . The number of position parameters is 32 including the abscissa and ordinate of each lamp. As the initial LED positions are generated by random, the SNR factor is over 100 in the beginning. As the initial LED positions are caused by uniform matrix distribution with 4 rows and 4 columns, the SNR factor is only 32.7. We set the maximum iteration number as 300000 . When the position optimization progress ends, the SNR factor turns 16.5. The irritation number of random initial positions is obviously more than matrix primary position in the same SNR factor.

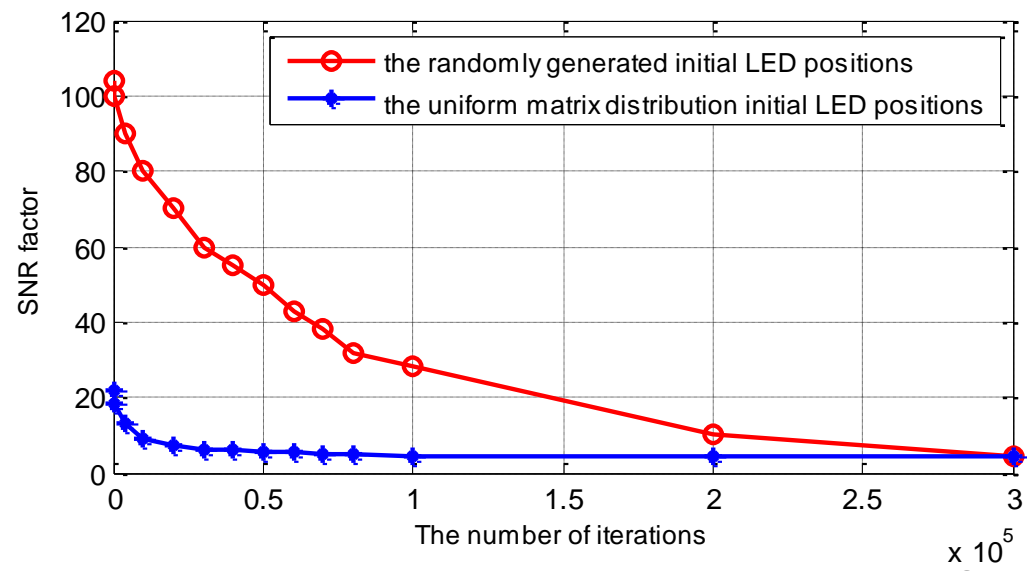

Figure 4. The Relation between the Iterations Number and SNR Factor of Fireworks Algorithm when N Equals 16

In the progress of optimization, five fireworks, fifty sparks, five Gaussian explosion sparks and the maximum iteration number 300000 are arranged. $x_{s i}$ and $y_{s i}$ range from 0 to 5 meters. We set a receiver point each 5 centimeters on the 0.85 meter high plane to accurately measure the SNR distribution in simulation calculation.

When the LED sources are arranged in the center of the room, the poor SNR distribution and contour lines on the receiver plane is shown as figure 5. The distance between user in the corner and the LED lamp is longer than the user in other position of the room. Therefore, the SNR of corner user is obviously lower than other users. The maximum SNR is 3034.8. The minimal SNR is 18.1270. The fluctuation of SNR on the receiver plane is 3016.6 with high volatility. The average SNR value is 565.6261. The SNR factor of the receiver plane is 118.545 .

Figure 6 is the SNR distribution and contour lines when LED-lamps are located separately and symmetrically as 4-by-4 matrix distribution. The SNR distribution 
gets improved for the separate lamp sources because the distances different between users and the LED sources are reduced.

The maximum SNR is 34.7998. The minimal SNR is 10.0307. The fluctuation of SNR on the receiver plane is 24.7691. The SNR average value is 26.9854. The SNR factor of the receiver plane is 21.5946 .
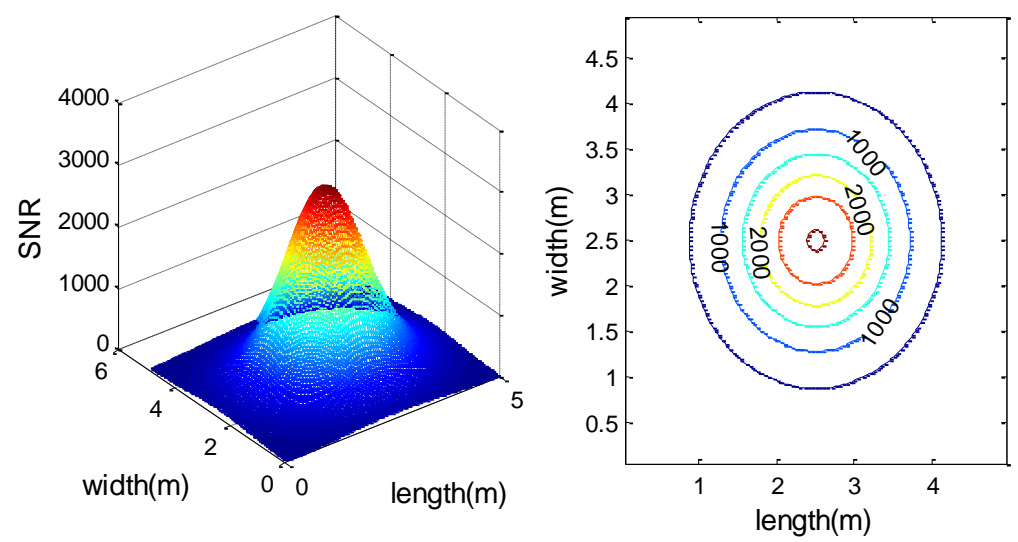

Figure 5. The SNR Distribution and Contour Lines when the LED Sources in the Center of the Room
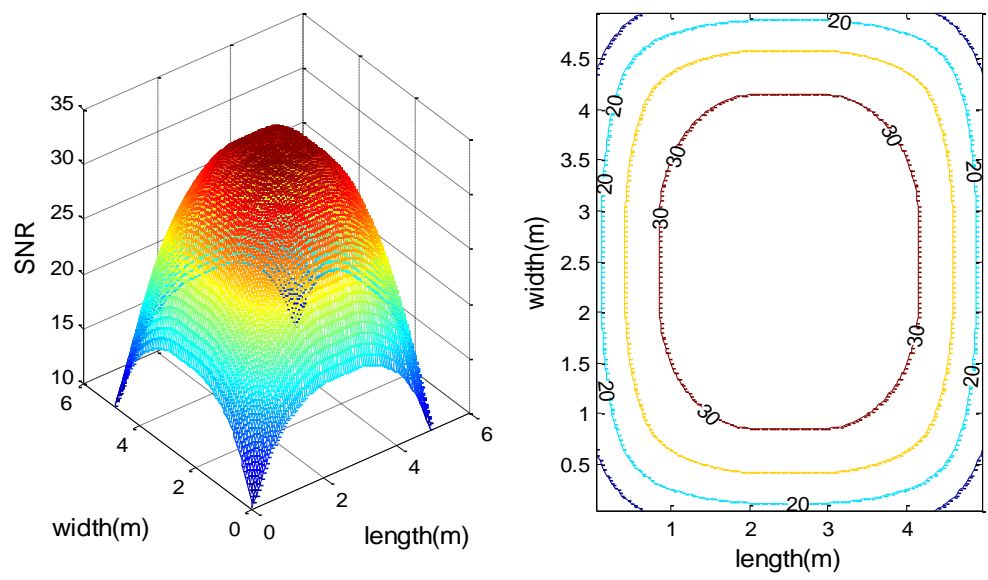

Figure 6. The SNR Distribution and Contour Lines when the LED Sources are Located Separately as 4-by-4 Matrix

We utilize 16 LED lamps as the signal sources in the room. After optimal computation, the positions of 16 LED lamps are shown as figure 7. Some LED sources are arranged nearly the edge of the room and some LEDS are arranged in the corner for increasing received power of the corner and edge. With this LED source arrangement, the SNR distribution is shown in figure 8. The maximum SNR is 22.1281. The minimal SNR is 15.1759. The fluctuation of SNR on the receiver plane is 6.9522. The SNR average value is 20.3975, The SNR factor of the receiver plane is 4.0885. With the optimization, the maximum SNR becomes smaller, the minimal SNR becomes bigger and the SNR fluctuation \& SNR factor significantly reduces. All of these mean the SNR distribution is more uniform. Note that, in the simulation we only consider the line of sight light but not think of the reflection of walls. Besides some special points in the corner of the room, the SNR of other positions are near 20 and the fluctuation is very small. With this method, we can get the almost uniform SNR distribution in most of light communication area. 


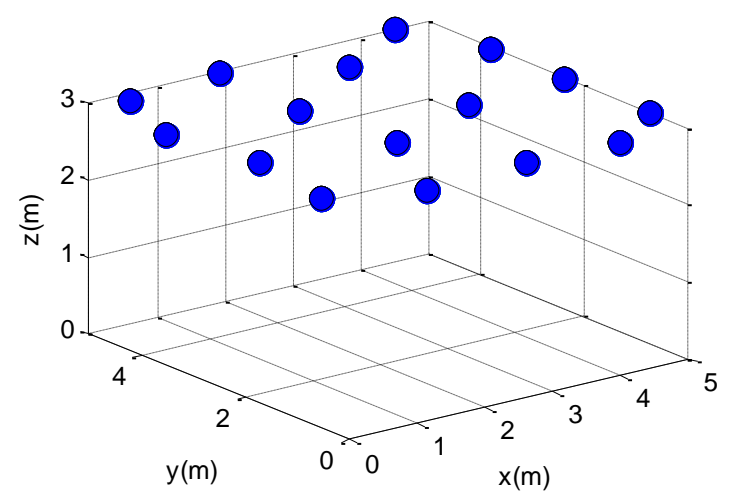

Figure 7. The Optimization Positions of $\mathbf{N}$ Lamps when $\mathbf{N}$ is $\mathbf{1 6}$
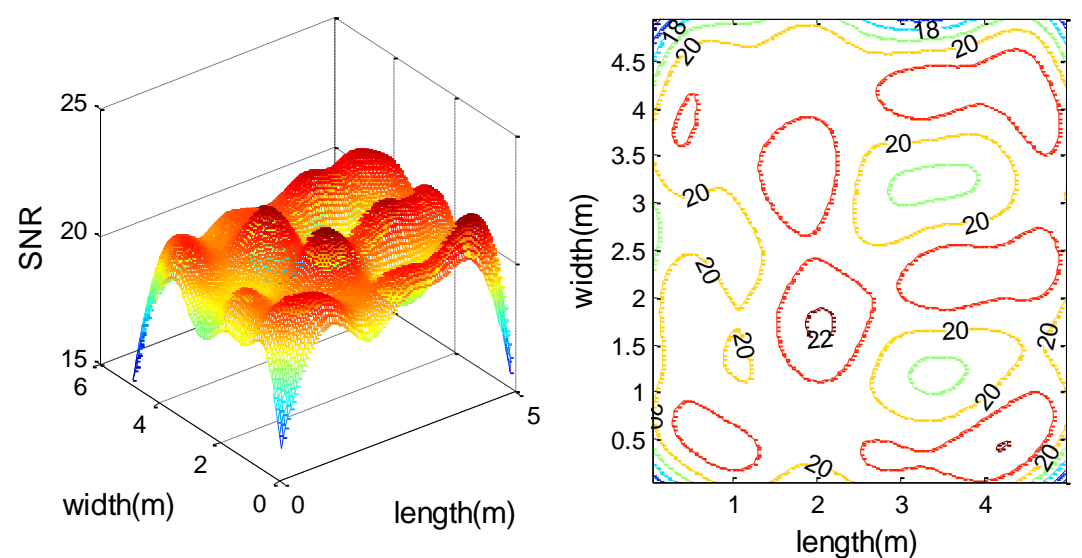

Figure 8. The SNR Distribution and Contour Lines of 16 LED Lamps with Optimal Position

When the lamp number is variable, this method can also be used to search optimal position of each lamp. In the next simulation, 9 LED lamps are utilized as signal sources in system. After the optimal simulation, the positions of 9 LED lamps are shown as figure 9. The SNR distribution is shown in figure 10 in this LED source arrangement. The maximum SNR is 50.33. The minimum SNR is 26.74. The fluctuation of SNR on the receiver plane is 23.59. The SNR average value is 40.51, The SNR factor of the receiver plane is 8.56. With this arrangement, the SNR distribution gets improved than lamps in the center of room. But the SNR distribution is worse than 16 LED lamps optimal arrangement.

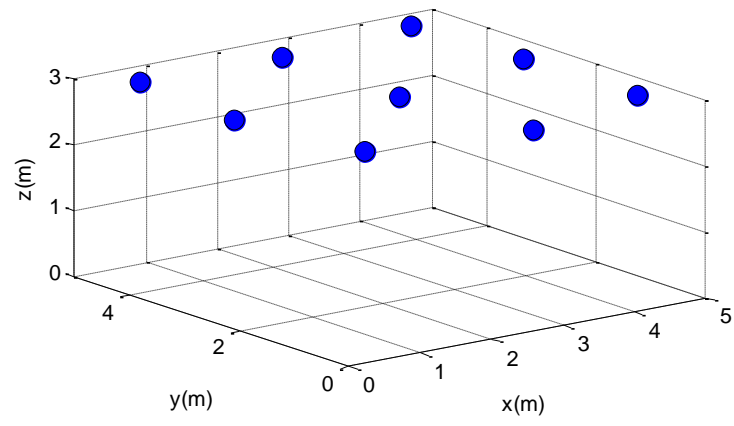

Figure 9. The Optimization Positions of $\mathbf{N}$ Lamps when $\mathbf{N}$ is 9 

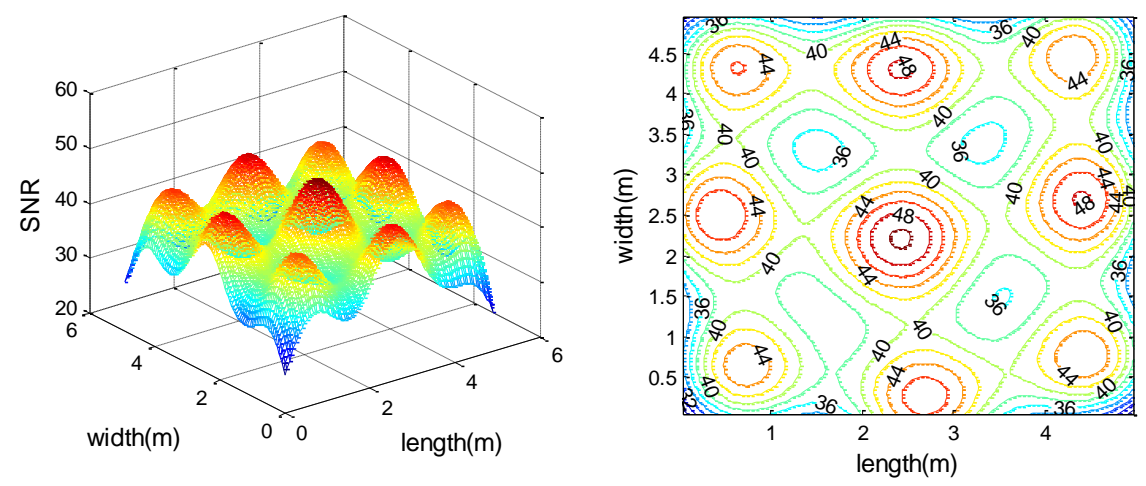

Figure 10. The SNR Distribution and Contour Lines of 16 LED Lamps with Optimal Position

In the following simulation, 4 LED lamps are used as signal sources in the VLC system. After the optimal simulation, the positions of 4 LED lamps are shown as figure 11. The SNR distribution is shown in figure 12 in this LED source arrangement. The maximum SNR is 199.23. The minimum SNR is 35.83. The fluctuation of SNR on the receiver plane is 163.4. The SNR average value is 115.55, The SNR factor of the receiver plane is 31.42.With this arrangement, the SNR distribution gets improved than lamps in the center of room. But the SNR distribution is worse than 16 and 9 LED lamps optimal arrangement. The more LED lamps used in system, the more uniform SNR distribution can get. And with this optimization method, all LED lamp optimal positions will be received by the firework algorithm no matter how many LED lamps number is requested in actual circumstance.

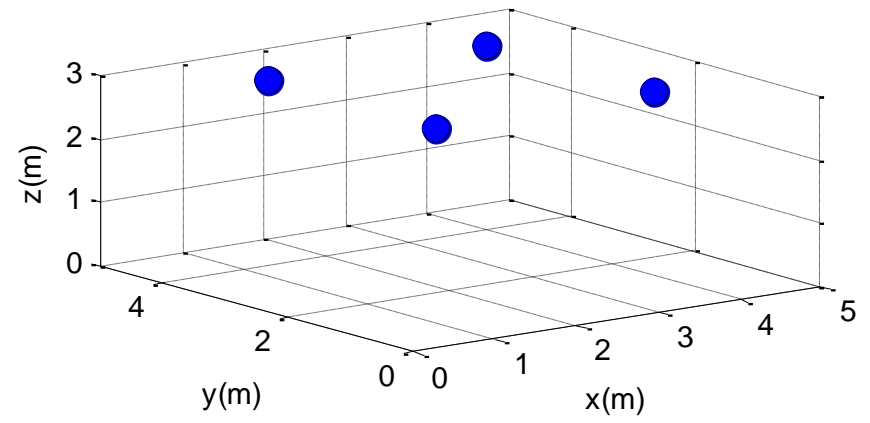

Figure 11. The Optimization Positions of $\mathbf{N}$ lamps when $\mathbf{N}$ is 4
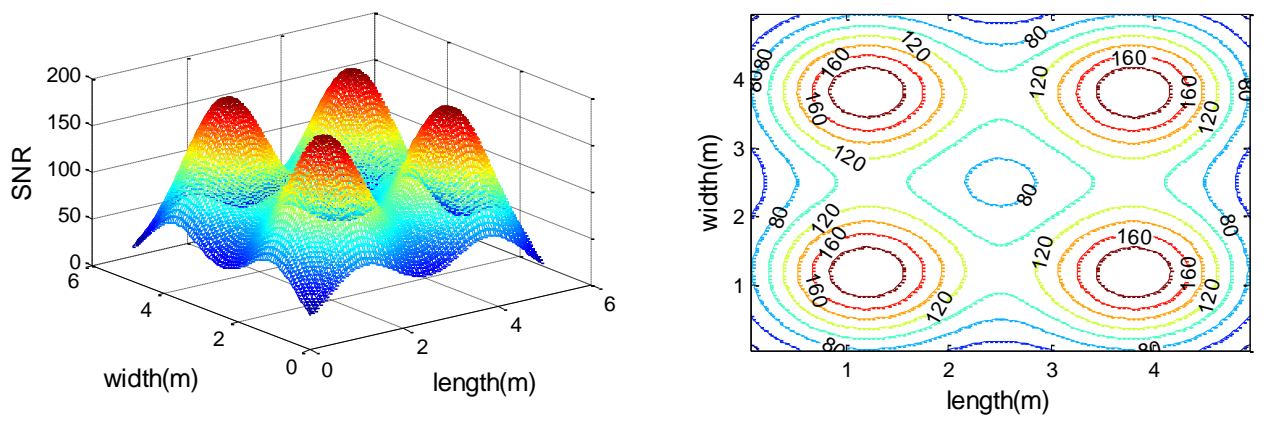

Figure 12. The SNR Distribution and Contour Lines of 4 LED Lamps with Optimization Position 


\section{Conclusion}

This paper investigates the performance of a novel LED lamp arrangement to reduce SNR fluctuation in indoor VLC communication space. The initial LED lamp arrangement consists of N LED lamps with $\sqrt{N}$ rows and $\sqrt{N}$ columns spreading evenly in the ceiling. All lamps are in the same height of 3 meters which is also the room's height. Based on the fireworks algorithm, we search the optimal LED positions in the ceiling to get the minimal SNR factor which represents the uniform SNR distribution on the receiver plane between different users. Our studies show that the SNR factor under such arrangement is obviously reduced, which guarantees that all users obtain almost identical communication quality, regardless of their locations. Moreover, we can find the optimal positions of all LED lamps and the best SNR distribution can be got when the number of LED lamp varies in some special situations. In reality, the number of LED lamps will be set for reaching your special SNR distribution demand. Our proposed optimal LED arrangement can give the best uniform performance to visible light communication system. When the LED lamps altitude can be regulated, this method can also be used to get the threedimension position of every lamp source. In addition, if the power of LED can be regulated, the optimal power of LEDs can be gotten by this method.

\section{Acknowledgements}

The authors gratefully acknowledge the support of the National Science \& technology Pillar Program of China (2013BAK06B05), the Nature Science Foundation of Jiangsu Province (BK20151148) and Postgraduate Training Innovation Project of Jiangsu Province (KYLX_1386).

\section{References}

[1] T. Komine and M. Nakagawa, "Fundamental analysis for visible-light communication system using LED lights", IEEE T Consum Electr, vol. 50, (2004), pp. 100-107.

[2] J. R. D. Retamal, H. M. Oubei, B. Janjua, Y. Chi, H. Wang, C. Tsai, T. K. Ng, D. Hsieh, H. Kuo, M. Alouini, J. He, G. Lin and B. S. Ooi, "4-Gbit/s visible light communication link based on 16-QAM OFDM transmission over remote phosphor-film converted white light by using blue laser diode", Opt Express, vol. 23, (2015), pp. 33656-33666.

[3] T. Mao, Z. Wang, Q. Wang and L. Dai, "Ellipse-based DCO-OFDM for visible light communications", Opt Commun, vol. 360, (2016), pp. 1-6.

[4] S. Prince and A. M. Vibin, "Optical Wireless Audio Communication Using LED Lighting System", Wireless Pers Commun, vol. 86, (2016), pp. 1159-1168.

[5] S. K. Routray, "The Changing Trends of Optical Communication", Potentials, IEEE, vol. 33, (2014), pp. 28-33.

[6] Z. Wang, C. Yu, W. Zhong, J. Chen and W. Chen, "Performance of a novel LED lamp arrangement to reduce SNR fluctuation for multi-user visible light communication systems", Opt Express, vol. 20, (2012), pp. 4564-4573.

[7] C. You, S. Zhang, Y. Zhai and Q. Hu, "New optimization method of LED lamp arrangement in mine work face for visual light communication", Journal of China University of Mining \& Technology, vol. 2, (2014), pp. 333-338.

[8] T. Komine and M. Nakagawa, "Integrated system of white LED visible-light communication and power-line communication", Consumer Electronics, IEEE Transactions, vol. 49, (2003), pp. 7179.

[9] Y. TAN, C. Yu, S. Zhang and K. Ding, "Introduction to Fireworks Algorithm", International Journal of Swarm Intelligence Research, vol. 4, no. 4, (2013), pp. 39-70.

[10] Y. Tan and Y. Zhu, "Fireworks algorithm for optimization", Adv Swarm Intell, vol. 6145, (2010), pp. 355-64.

[11] A. M. Imran and M. Kowsalya, "A new power system reconfiguration scheme for power loss minimization and voltage profile enhancement using Fireworks Algorithm", International Journal of Electrical Power \& Energy Systems, vol. 62, (2014), pp. 312-322.

[12] Y. Zheng, Q. Song and S. Chen, "Multiobjective fireworks optimization for variable-rate fertilization in oil crop production", Appl Soft Comput, vol. 13, (2013), pp. 4253-63. 


\section{Authors}
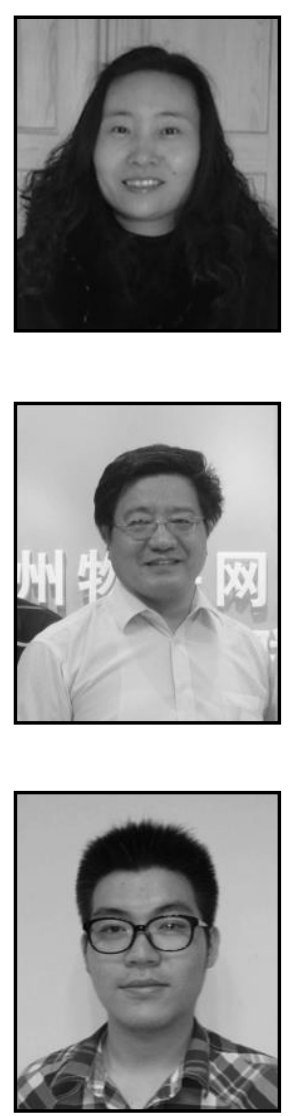

Chunxia You received the B.S. degree from School of Physics and Electronic Engineering, Jiangsu Normal University, Xuzhou, China in 2001, and the M.S. degree from School of Information and Electrical Engineering, China University of Mining \& Technology, Xuzhou, China in 2007. Currently, she is working toward the Ph.D. degree at China University of Mining \& Technology. Her research interests include visible light communications and signal processing for communications.

Shen Zhang received the B.S. degree from Department of Radio Engineering, Zhejiang University, Hangzhou, China in 1982 and the Ph.D. degree from China University of Mining \& Technology in 2001. Currently, he is a Full Professor in the Internet of Things Research Center, China University of Mining and Technology. His current research interests include mine communication and information technology.

Ran Zhang received the B.S. degree from School of Information and Electrical Engineering, China University of Mining \& Technology, Xuzhou, China in 2012. Now, he is working for the Ph.D. degree in China University of Mining \& Technology. His research interests include compressed sensing and precision time synchronization. 